\title{
Research on Accelerated Degradation Test of RV Reducer
}

\author{
Jianghua Ge, Bo Lu, Yaping Wang and Xudong Jiang \\ School of Mechanical Engineering, Harbin University of Science and Technology, \\ Harbin, 150080, China \\ 785063338@qq.com
}

\begin{abstract}
In view of the shortcomings of traditional accelerated life testing in the process of nonfailure test data, Accelerated degradation test of $R V$ reducer is carried out in this paper. Due to the special structure and working principle of $R V$ reducer, it is very difficult to estimate the life of the problem quickly and effectively in practical work, so this paper made the accelerated degradation test to solve these problems. Firstly we analysis the failure mechanism of RV Reducer, select the motor torque as the accelerated stress, list scheme of accelerated degradation test and obtain test data through practical test, establish accelerated degradation model, get reliability index of $R V$ reducer with the method of statistic and predict the life. The above results show that this method can not only save time and cost, but also greatly reduce the experimental period and provide a basis for predicting the life of $R V$ Reducer.
\end{abstract}

Keywords: RV Reducer, Accelerated Stress, Reliability, Accelerated Degradation Test.

\section{Introduction}

$\mathrm{RV}$ reducer, a kind of two stage reducer based on the type of the pin, has been widely used in recent years by experts and scholars [1]. This is a kind of RV reducer shown in Figure 1. RV reducer has many advantages compared with the traditional planetary gear reducer. For example small size, light weight, strong load capacity, high rigidity, long service life, high transmission efficiency, stable transmission process, high movement precision and small rotation error [2]. RV reducer has been widely used in the field of industrial robot and so on.

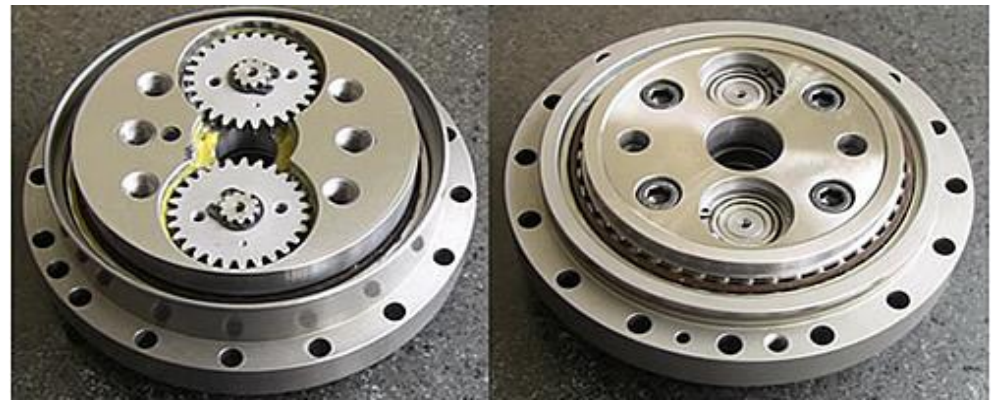

Figure 1. RV Reducer

Due to the heavy load at work, the reliability of its work must also be strictly required as the core component of industrial robot [3]. Unit price of ordinary RV reducer can reach up to tens of thousands of yuan. It will bring great trouble to the maintenance, security and even huge economic burden when it suddenly damage during the work. So it is very important to accelerate the degradation test of RV reducer. In this paper through the accelerated degradation, we can get the actual life of the reducer in a relatively short 
period of time. It can bring great convenience and protection to product maintenance and repair.

\section{Failure Cause of RV Reducer}

Before the accelerated degradation test, we must firstly analyze the fundamental reason clearly for the failure of RV reducer. First of all RV reducer always has high structural strength and it is often used in low speed situations, so it is difficult to occur to mechanical failure of internal members under normal working conditions. Most of the failures are wear failure [4]. Wear generally comes from the wear between the crank shaft and the gear and the wear between the needle and the cycloid gear. At the same time there is also the exact experimental data to prove it as shown in Table 1. Table 1 shows wear is the main failure mode whether it is the bearings or gears. So we think that the reason for the failure of the gear reducer is wear. However we also need to take into account the temperature, external load, speed and other non-decisive factors.

Table 1. Invalid Form Data of Bearings and Gears

\begin{tabular}{|c|c|c|}
\hline Failure mechanism & Bearing/\% & Gear/\% \\
\hline Wear & 85 & 65 \\
\hline Fatigue & 1 & 15 \\
\hline Collision & 2 & 20 \\
\hline Corrosion & 12 & - \\
\hline
\end{tabular}

\section{Accelerated Test Method of RV Reducer}

There are many ways to speed up the loading stress. The stress can be divided into three types by the test load [5], they are Constant-Stress, Step-Stress and ProgressiveStress. The diagrams of them are shown in Figure 2.

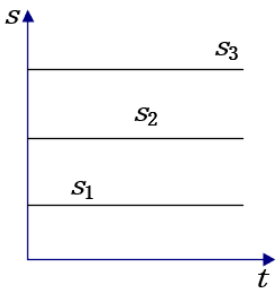

(a)

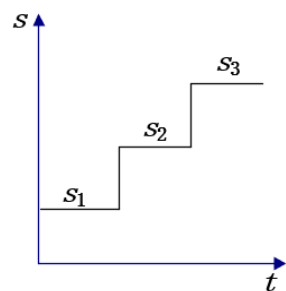

(b)

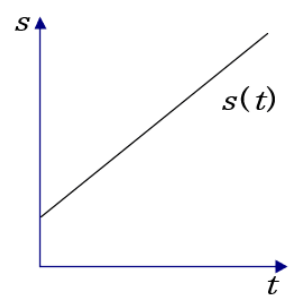

(c)

Figure 2. Common Acceleration Stress

Among these circumstances, Analysis of test data is relatively simple for ConstantStress. And its result is more accurate. Test sample of Step-Stress is less than others, but the result is not as accurate as Constant-Stress. Progressive-Stress has a high demand for equipment, is not suitable for the actual situation. So this paper chooses Constant-Stress.

\subsection{Accelerated Stress Selection}

(1) Selecting the temperature as the acceleration stress

Based on the obtained conditions, both between the gear and the crank shaft but also and between the cycloid gear and needle use lubrication technology named Braycote 601 . The viscosity reaches nearly 400 . If the outside temperature is very small, there is little effect on the lubrication of RV reducer. So it also cannot achieve the purpose of 
accelerated wear. If increasing the temperature substantially, it will lead to change the reason of the failure of the product. This does not meet the purpose of accelerated degradation test. So this method is not used in this paper.

(2) Selecting the speed as the acceleration stress

If we choose the right speed, RV reducer has been greatly improved of its lubrication situation when selecting the rotational speed as the acceleration stress. However this method will increase roller wear and reduce the efficiency.

Integrating above situation, this paper chooses to increase the torque as the acceleration stress.

\subsection{Accelerated Degradation Test Procedure}

(1) Choosing to increase the torque as the acceleration stress, increasing the torque value according to the geometric law and begin the experiment;

(2) We randomly select 15 random samples, do accelerated life test under 3 stress levels, three stress levels were $T_{1}=10 \mathrm{n} . \mathrm{m}, T_{2}=20 \mathrm{n} . \mathrm{m}$ and $T_{3}=30 \mathrm{n} \cdot \mathrm{m}$.

(3) 15 random samples are divided into three groups, 5 samples are tested for a group. Selecting a number of time points to measure and recorded the performance degradation of the sample.;

(4) During the Acceleration test, the others including temperature, lubrication conditions, speed and other objective factors are all the same in addition to the applied torque;

(5) The life of RV reducer is quite long and objective conditions do not allow for long periods of time. So we think it failed when the transmission efficiency of RV reducer reduced to $50 \%$ during the Acceleration test. At this time we stopped testing. In other words we thought failure threshold of RV Reducer $D_{f}=50$.

\section{Test Data Processing}

We finished the Accelerated degradation test according to the above requirement, test ed the date and established the accelerated degradation model of RV reducer. Finally the reliability and service life of RV reducer were predicted by the method of statistical analysis.

\subsection{Typical Acceleration Model}

(1) Arrhenius model [6]

In accelerated degradation test the most common acceleration stress is often temperature. Increasing temperature will make the electronic components, insulation materials produced by chemical reactions. It will accelerate the failure process of the product. Its acceleration mathematical model is shown as

$$
\eta=A \exp \left[E_{a} /(k T)\right]
$$

Where $\mathrm{T}$ is accelerated stress level of temperature, $\eta$ is the life characteristic of the product under the action of temperature accelerated stress $\mathrm{S}, \mathrm{A}$ is the constant associated with other failure factors, $E_{a}$ is activation energy, it relates to the product of the material, $\mathrm{K}$ is Boltzmann constant.

(2) Inverse power law model [7]

Inverse power law model often describes relationship between the characteristics of the product life and the level when the mechanical stress or electrical stress is the acceleration stress. Its acceleration mathematical model is shown as 


$$
\eta=A S^{\gamma_{1}}
$$

Where $\mathrm{S}$ is accelerated stress level, $r_{1}$ constants associated with failure modes. SN curve [8] is a curve that describes the relationship between fatigue life and cyclic loading of metallic materials. SN curve is one of the most common forms of inverse power law.

(3) Eyring model

We often use Eyring as an accelerated life model when two different stresses are used as the acceleration stress and one of the accelerated stress is temperature. Its acceleration mathematical model is shown as

$$
\eta=(A / T) \exp [B /(k T)] \cdot \exp \{V[C+D /(k T)]\}
$$

Where A B C D is measured by the accelerated life test., $\mathrm{T} \mathrm{V}$ are two kinds of accelerated stress levels.

(4) Polynomial acceleration model

The first three are typical accelerated life models. They all can be realized by means of a certain mathematical method. In some cases, it is impossible to achieve linearization. Then we will use the polynomial acceleration model. Its acceleration mathematical model is shown as

$$
\ln \eta=\gamma_{0}+r_{1} \varphi(S)+r_{2}[\varphi(S)]^{2}+\cdots+r_{k}[\varphi(S)]^{k}
$$

Polynomial acceleration model is seldom used in general. When the life model is not linear, this means that its accuracy may not meet the requirements. And there is a greater risk of error of the data fitting degree for the mathematical model. So we will choose several acceleration models in general.

\subsection{Test Data Analysis}

After the accelerated degradation test is done, we can get a series of test data through the testing device. The test data is shown in Table 2. Table 2 shows rotation times of RV reducer under three kinds of Accelerating Stress before RV reducer transmission efficiency is less than $50 \%$. From the table we can clearly find that choosing the torque as the acceleration stress does play a great role, rotation of different RV reducer is quite different from each other before failure under the condition of different acceleration stress.

Table 2. Rotation Times of RV Reducer under Different Stress

\begin{tabular}{|c|c|c|c|c|c|}
\hline & \multicolumn{5}{|c|}{ Sample number } \\
\hline $\begin{array}{c}\text { Accelerated } \\
\text { stress }\end{array}$ & 1 & 2 & 3 & 4 & 5 \\
\hline $10 \mathrm{n} . \mathrm{m}$ & $8.18 \times 10^{6} r$ & $8.45 \times 10^{6} r$ & $8.24 \times 10^{6} r$ & $8.12 \times 10^{6} r$ & $8.76 \times 10^{6} r$ \\
\hline $20 \mathrm{n} . \mathrm{m}$ & $5.18 \times 10^{6} r$ & $5.46 \times 10^{6} r$ & $5.48 \times 10^{6} r$ & $5.71 \times 10^{6} r$ & $5.02 \times 10^{6} r$ \\
\hline 30n.m & $3.78 \times 10^{6} r$ & $3.46 \times 10^{6} r$ & $3.72 \times 10^{6} r$ & $3.12 \times 10^{6} r$ & $3.80 \times 10^{6} r$ \\
\hline
\end{tabular}

In the process of accelerated degradation test, we will have a period of time for each sample data collection, analysis of performance degradation, and finish records until single sample performance degradation to the threshold then we should stop the test. Then we extract 3 samples under different stress conditions and use MATLAB to synthesize the curves. Their curves were shown in Figure 3. We can clearly show that the performance of the RV reducer is in a declining trend from Figure 3. The only difference is that the performance degradation of the speed. At the same time we can also note that the three 
curves show a monotonic decreasing trend. The accord with the principle of accelerated degradation has been declined.
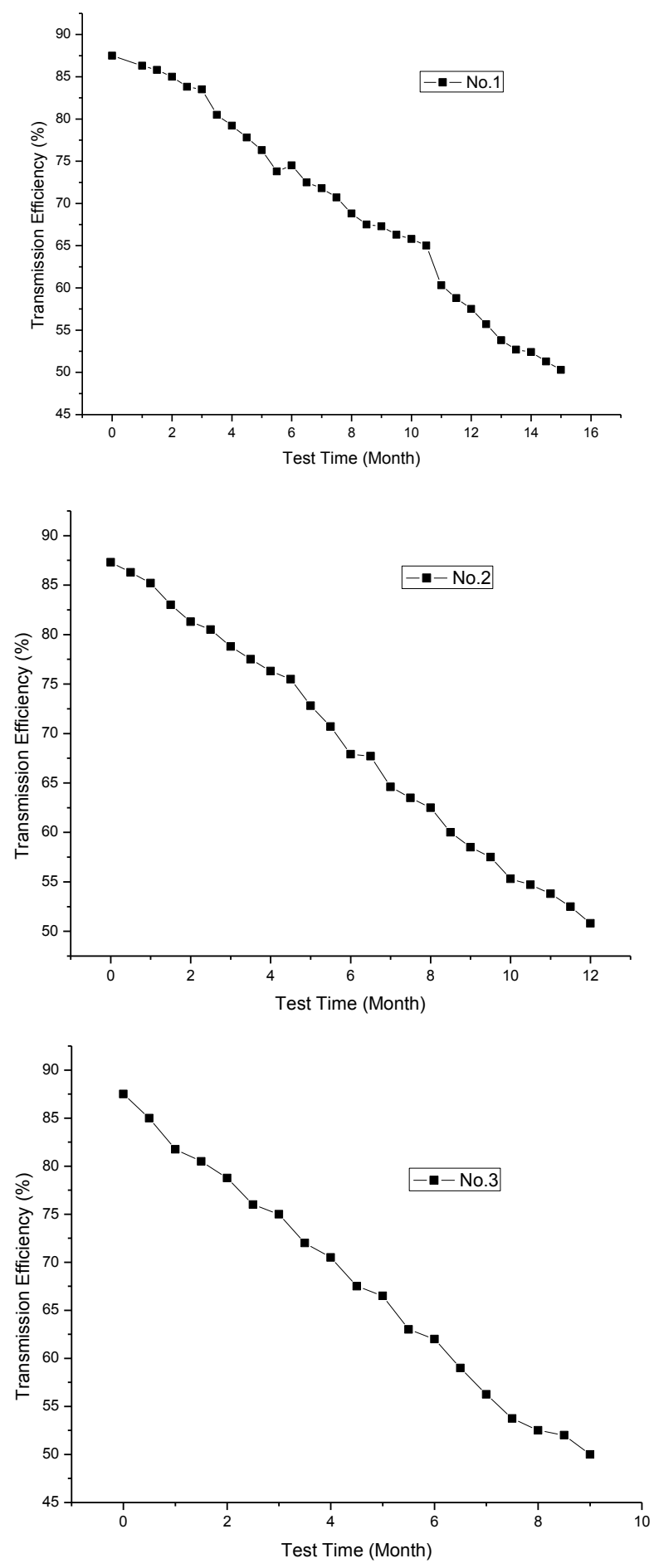

Figure 3. Three Kinds of Degradation Curves under Different Stress

After completing all the samples of accelerated degradation test, we speed up the test data in the form of a table as shown in Table 3. Since the test process will be recorded once every period of time, so that all the data cannot be displayed in the chart, so this paper selected a number of representative time points recorded in the chart. 
Table 3. Accelerated Degradation Test Data of RV Reducer

\begin{tabular}{|c|c|c|c|c|c|c|}
\hline $\begin{array}{c}\text { Accelerated } \\
\text { stress }\end{array}$ & $\begin{array}{c}\text { Sample } \\
\text { number }\end{array}$ & \multicolumn{4}{|c|}{ Measuring time (month) and Degradation percentage\% } \\
\cline { 2 - 7 } & 1 & 2 & 4 & 6 & 7 & 9 \\
\hline \multirow{4}{*}{ 10n.m } & 2 & 3.46 & 7.56 & 10.56 & 15.78 & 22.75 \\
\cline { 2 - 7 } & 3 & 2.94 & 7.45 & 11.45 & 14.78 & 21.54 \\
\cline { 2 - 7 } & 4 & 2.87 & 6.45 & 13.45 & 15.75 & 19.78 \\
\cline { 2 - 7 } & 5 & 3.56 & 5.45 & 10.45 & 16.45 & 19.45 \\
\hline \multirow{4}{*}{ 20n.m } & 6 & 5.78 & 10.56 & 15.45 & 24.78 & 31.45 \\
\cline { 2 - 7 } & 7 & 4.88 & 11.45 & 17.45 & 21.78 & 29.74 \\
\cline { 2 - 7 } & 8 & 5.71 & 12.45 & 16.45 & 20.64 & 33.45 \\
\cline { 2 - 7 } & 9 & 5.05 & 9.45 & 15.78 & 22.45 & 32.78 \\
\hline \multirow{4}{*}{30 n.m } & 10 & 4.79 & 10.45 & 14.45 & 19.56 & 30.78 \\
\cline { 2 - 7 } & 11 & 10.46 & 18.45 & 25.78 & 35.78 & 50.46 \\
\cline { 2 - 7 } & 12 & 10.71 & 19.45 & 27.45 & 30.45 & 55.13 \\
\cline { 2 - 7 } & 13 & 10.56 & 17.65 & 26.45 & 33.45 & 51.56 \\
\cline { 2 - 7 } & 14 & 10.78 & 20.45 & 24.54 & 29.78 & 49.46 \\
\hline
\end{tabular}

\subsection{Statistical Analysis of Product Performance Parameters}

The performance degradation of RV reducer has the characteristics of continuous and independent increment. So the degradation model of RV reducer can be listed on the basis of the Brown movement [9]. The Brown movement itself represents the independence of individual movement. So the accelerated degradation model can be understood as the cumulative effect of the Brown motion degradation process. Its acceleration mathematical model is shown as

$$
X(t)=X_{0}+a(t)+\sigma W(t)
$$

Where $X_{0}$ is the initial degradation value of the production, $a(t)$ is drift term, $\sigma$ is Constant number, $W(t)$ is the standard of Brown campaign, $W(t) \sim N(0, t)$.

In this paper, we list the acceleration degradation model of RV reducer. Its acceleration mathematical model is shown as

$$
Y(t)=Y_{0}+M(s) t+\sigma W(t)
$$

Where $Y(t)$ is the performance of the reducer, $Y_{0}$ is initial degradation, $W(t)$ is the standard Brown campaign, $\sigma$ is diffusion coefficient, it is used to describe the difference between the product and the effect of external factors on the product, $M(s)$ is degradation coefficient, here is a constant because we are using a constant stress accelerated test.

Because the movement properties of the Brown movement belong to Markov process effect [10], the increment in the process of accelerated degradation test is independent of each other(Markov process effect: As known in the present case, its future evolution does not depend on the past). Because the Brown movement itself belong to normal distribution, degenerate delta $\left(Y_{i}-Y_{i-1}\right)$ is also subject to normal distribution, its mean value is $\mu(T) \Delta t$, its variance is $\sigma^{2} \Delta t$, its probability density is shown as

$$
f_{i, i-1}=\frac{1}{\sigma \sqrt{\Delta t}} \varphi\left[\frac{\left(Y_{i}-Y_{i-1}\right)-\mu \Delta t}{\sigma \sqrt{\Delta t}}\right]
$$


Where $\varphi$ is Function of standard normal distribution probability density.

Failure threshold is the critical value of human identification. This proves that the product has failed when the performance of the product reduced to below the threshold value. In accordance with the understanding of the Brown movement is a time that one point reaches the point of failure. The distribution of the first time of the Brown movement follows the inverse Gauss distribution. Its probability density function has been shown as

$$
f\left(t ; Y_{0}, C\right)=\frac{C-Y_{0}}{\sqrt{2 \Pi \sigma^{2} t^{3}}} \exp \left\{-\frac{\left[\left(C-Y_{0}\right)-\mu \cdot t\right]}{2 \sigma^{2} t}\right\}
$$

Where $\mathrm{C}$ is performance of critical value

So after mathematical derivation can get the RV reducer reliability function, it is shown as

$$
R(t)=\Phi\left[\frac{\left(C-Y_{0}\right)-\mu \cdot t}{\sigma \sqrt{t}}\right]-\exp \left[\frac{2 \mu\left(C-Y_{0}\right)}{\sigma^{2}}\right] \Phi\left[-\frac{\left(C-Y_{0}\right)+\mu \cdot t}{\sigma \sqrt{t}}\right]
$$

We can clearly find from reliability function, we can get the reliable life, reliabili-ty and other indicators of the RV reducer as soon as we know $\mathrm{C} \mu \sigma$. If we want to get the date, then we have to solve the data obtained through the experimental proc-ess.

We assume we selected $\mathrm{m}$ test samples in accelerated degradation test and we measured $\mathrm{n}$ times, the resulting data will form a matrix M. It is shown as

$$
M=\left|\begin{array}{cccc}
D_{11} & D_{12} & \cdots & D_{1 n} \\
D_{21} & D_{22} & \cdots & D_{2 n} \\
\vdots & \vdots & & \vdots \\
D_{m 1} & D_{m 2} & \cdots & D_{m n}
\end{array}\right|
$$

Any element within the matrix $D_{i j}$ represents the performance degradation data obtained by the first $\mathrm{RV} i$ reducer of the first $j$ test. $D_{i 1}$ represents the first measurement of performance degradation of the first sample, then the resulting matrix can be further degraded by the performance degradation of the RV reducer. It is shown as

$$
\Delta M=\left|\begin{array}{cccc}
D_{11}-D_{12} & D_{11}-D_{13} & \cdots & D_{11}-D_{1 n} \\
D_{21}-D_{22} & D_{21}-D_{23} & \cdots & D_{21}-D_{2 n} \\
\vdots & \vdots & & \vdots \\
D_{m 1}-D_{m 2} & D_{m 1}-D_{m 3} & \cdots & D_{m 1}-D_{m n}
\end{array}\right|
$$

Now we use the elements from $\Delta M$, and use the maximum likelihood estimation Error! Reference source not found. of $\mu$ and $\sigma$ parameter estimation. Its likelihood function is shown as

$$
L(\sigma, \mu)=\prod_{i=1}^{m} \prod_{j=1}^{n} \frac{1}{\sigma \sqrt{2 \Pi \cdot \Delta t}} \exp \left[-\frac{\left(\Delta D_{i j}-\mu \Delta t\right)^{2}}{2 \sigma^{2} \cdot \Delta t}\right]
$$

According to the maximum likelihood estimation algorithm, a set of equations is shown as 


$$
\left\{\begin{array}{l}
\frac{\partial \ln L(\mu, \sigma)}{\partial \mu}=0 \\
\frac{\partial \ln L(\mu, \sigma)}{\partial \sigma}=0
\end{array}\right.
$$

Finally we derive the maximum likelihood estimate of the parameters $\mu$ and $\sigma$, it is shown as

$$
\left\{\begin{array}{l}
\hat{\mu}=\frac{\sum_{i=1}^{m} \sum_{j=1}^{n} \Delta D_{i j}}{m n \Delta t} \\
\hat{\sigma}=\sqrt{\frac{\sum_{i=1}^{m} \sum_{j=1}^{n}\left(\Delta D_{i j}-\mu \Delta t\right)^{2}}{m n \Delta t}}
\end{array}\right.
$$

Now we get all the product parameters and all bring $\mu \sigma \mathrm{C}$ into the formula 9, then the reliability estimates and life parameters of the RV reducer can be obtained.

\section{Summary}

In this paper we studied the accelerated degradation test of RV reducer. Research shows that wear is the main failure of RV reducer. We choose torque as the acceleration stress and select the appropriate span of the torque as the input of the test. We obtained the performance degradation images of samples under different torque conditions after the experiment, then choosing appropriate method to establish the accelerated degradation model of RV reducer. Finally we can predict the reliability and normal life of the product with the method of statistical analysis. This method can shorten the test cycle, save test cost and provide some practical reference value for the life prediction of RV reducer.

\section{Acknowledgements}

This paper is supported by University Science Park Entrepreneurial Talent of Innovation Talents of Science and Technology of Harbin Application Technology Research and Development Project (51575143).

\section{References}

[1] L.L. Xing, H.W. Dong, W.X. Qi, L.C. Bo, W.Z. Wei and F. Rong, "Research on high precision RV drive for robot", Journal of Dalian Railway Institute, vol. 02, (1999), pp. 1-11.

[2] Z. Jing, "Industrial Robot Reducer RV320: Modification Parameters Optimization and Processing Research", Tianjin University, (2013).

[3] Z.L. Lin, "Analysis on the dynamic characteristic of RV reducer", Henan University of Science and Technology, (2015).

[4] Z.F. Shou, Z.L. Lin, L.J. Ting and Z. Peng, "Review of the Study on Dynamic Characteristic of RV Reducer", Journal of Mechanical Transmission, vol. 08, (2014), pp. 174-176.

[5] L.J. Yang, "Failure Mechanism Theory and Accelerated Life Testing Method Research for Space Lubrication Harmonic Drive", Chongqing University, (2012).

[6] C. Hong, "Study on Accelerated Life Testing of the Filtering Gear Reducer under Space Environment", University of Electronic Science and Technology of China, (2014).

[7] Y.Y. Long, "Research on Life Prediction for Rolling Bearing based on Accelerated Life Testing", National University of Defense Technology, (2012).

[8] M. Yong, "Extreme Maximum Model and Application for the Probabilistic Fatigue S-N Relations", Southwest Jiaotong University, (2003).

[9] R.S. Hong, Z.H. Fu and B. Fang, "Real-time performance reliability prediction for civil aviation engines based on Brownian motion with drift", Journal of Aerospace Power, vol. 12, (2009), pp. 2796-2801.

[10] X.R. Ting, "Prediction of Markov fuzzy reliability for existing bridge", Changan University, (2006).

[11] Z.C. Yi, L. Cheng, F.C. Wei, H.G. Ping and S.X. Dong, "Reliability analysis of aero engine blade with extreme response surface method", Harbin University of Science and Technology, (2005). 